ITC $1 / 50$

Information Technology and Control

Vol. 50 / No. 1/ 2021

pp. 171-187

DOI 10.5755/j01.itc.50.1.27494
EECCRN: Energy Enhancement with CSS Approach Using Q-Learning and Coalition Game Modelling in CRN

\begin{tabular}{|c|c|}
\hline Received 2020/08/14 & Accepted after revision 2021/10/27 \\
\hline Cross & http://dx.doi.org/10.5755/j01.itc.50.1.27494 \\
\hline
\end{tabular}

HOW TO CITE: Shanmuganathan, V., Kalaivani, L., Kadry, S., Suresh, A., Robinson, Y. H., Lim, S. (2021). EECCRN: Energy Enhancement with CSS Approach Using Q-Learning and Coalition Game Modelling in CRN. Information Technology and Control, 50(1), 171-187. https://doi.org/10.5755/j01.itc.50.1.27494

\title{
EECCRN: Energy Enhancement with CSS Approach Using Q-Learning and Coalition Game Modelling in CRN
}

\section{Vimal Shanmuganathan}

Department of CSE, Ramco Institute of Technology, Rajapalayam, Tamil Nadu, India; e-mail: svimalphd@gmail.com

\section{Kalaivani}

Department of EEE, National Engineering College, Kovilpatti, Tamil Nadu, India; e-mail: anuprakad@gmail.com

\section{Seifedine Kadry}

Faculty of Applied Computing and Technology, Noroff University College, Kristiansand, Norway; email: skadry@gmail.com

\section{A. Suresh}

Department of CSE, SRM Institute of Science \& Technology, Kattankulathur, Tamilnadu, India; e-mail:prisu6esh@gmail.com

\section{Y. Harold Robinson}

School of Information Technology and Engineering, Vellore Institute of Technology, Vellore, India; e-mail:yhrobinphd@gmail.com

\section{Sangsoon Lim}

Department of Computer Engineering, Sungkyul University, Anyang, South Korea; e-mail:lssgood80@gmail.com 
The Cognitive radio network (CR) is a widespread technology in which the Secondary users are engaged in acquiring the idle spectrum from the primary user. The secondary users with the help of Spectrum monitoring agents acquire the spectrum to avoid the false alarm possibility. The collaborative spectrum sensing (CSS) is an approach that will identify the false intruder in the CR networks, here it is proposed with the Enhanced Q-Learning model with Coalition Game approach (EQLCG) to outline the energy enhancement. Besides an approach on Greedy Bidding is used to allocate the spectrum to the winning secondary user (SU) based on the idle primary user to strengthen the spectrum sensing. The winning secondary user forms a communication establishment with the neighbouring SU to eradicate the miss detection probability based on group level cooperation. The simulation experiment analyses the cluster level security with energy monitoring that has been performed using the analysis of interference by applying the coalition game theory modelling and the information obscured by the attacker is reduced with the usage of enhanced Q-learning, and the results prove that overhead is substantially monitored. The proposed paper enhances the security in physical layer with energy conservation and maintains the spectrum usage for application purpose. The proposed simulation approach reduces the miss detection and false alarm probabilistic approach while compared with Stackelberg and Bayesian game models.

KEYWORDS: CR networks, Physical Layer Security, Coalition Game Theory, Spectrum Sensing, Q-Learning.

\section{Introduction}

The demand in wireless communication has emerged widespread in the spectrum management and spectrum handling market. In the modern era, wireless devices occupy a marketable solution for all sources of data communication. Security is a major concern, even though a lot of applications came into existence. Besides their geographical position, wireless communication makes the information sharing and accessing of the information in the global era at ease. Wireless communication can be classified as an infrastructure network and infrastructure-less networks. The wireless communications perform a key role in the modern era for making efficient communication; besides CR networks have been best known for the spectrum allocation in the modern digital world. The spectrum allocation and the spectrum utilization occupy a major impact in the wireless communication to support the demand in the increase of the spectrum utilization [31]. The CR networks are utilized with the available spectrum bands, where the unlicensed users that are secondary users (SU) and the licensed users that are primary users (PU) utilize the spectrum to avoid interference in the physical layer communication. . Each secondary user attempts the idle spectrum to utilize it with the support of the primary agents residing in the same cluster of SU, and hence the utilization may be increased parallel in the CR networks. The Spectrum sensing is done by the SU that analyze the spectrum for the best efficient usage, and hence the accuracy is enriched that makes the spectrum allocation and sensing parameter to be estimated well [32].

The CR networks may be susceptible to various factors that affect the spectrum allocation and the utilization such as propagation loss, channel fading, Misdetection ratio in a higher altitude, and channel noise [47]. The spectrum sensing may be enriched to avoid the false alarm and miss detection. The false alarm probability is a measure, where the $\mathrm{SU}$ assumes that the spectrum is busy with the PU even when the spectrum is idle, it leads to the false alarm probability and intruder to enter in to the CR network [35]. The miss detection is a case, where the spectrum is assumed to be idle even when it has been used by some other PU. The Miss detection and false alarm are the sensing errors that often happened in CR networks in a high probability compared to the other authentication approaches. These sensing errors in SU may be a big barrier to the user allocation within the SU and also the utilization of the spectrum with the PU [48]. This spectrum sensing approach restricts the miss classification when the SU is in large number and focus on the winning allocation, and it may variably increase the physical communication in the network due to the selection of the best SU among the cluster from the greedy bidding, in such cases, the miss classification is avoided by the configured $\mathrm{SU}[40]$. The second frame is that the winning SU must increase the idle spectrum detection ratio that may increase the communication paradigm [2].

The spectrum sensing is proposed herewith in a collaborative manner termed as collaborative spectrum sensing, where the accuracy is enriched in the collab- 
orative spectrum sensing [29]. The CSS approach collects the sensing report with the support of the fusion centre, and the centre prepares a collective sensing report in a cooperative manner [14]. The cooperative spectrum sensing approach has been implemented in SU to enhance the spectrum sensing, where it will provide a good authentication mechanism compared to the normal sensing, and it actuates the secrecy rate to avoid jamming and interference with allowing only the authenticated user to acquire the spectrum. The coalition game theory approach has been applied with the CSS to investigate the interference analysis between the PU and SU [45]. The miss detection probability is assumed to be reduced with the coalition game approach, where the intruder will maliciously intrude in the $\mathrm{CR}$ network by advertising them as $\mathrm{SU}$, and the interference analysis within $\mathrm{PU}$ is done at the miniature level with this game approach. The communication between neighbouring $\mathrm{SU}$ is increased with the PU outside the cluster to avoid miss detection [5]. Figure 1 depicts the usage of SU with group formation, and the winning SU from the spectrum allocated group by the PU are formed in a group with SU1 to SU5, and the losing group informs the SU during transmission and the time slot allocation performs the miss detection probability [26].

Figure 1 depicts the proposed model of selecting the winning SU, and a group has been formed with these winners. The Primary User 1 connects with the SU1 and SU5 for accumulating the usage of spectrum access. The PU2 has its spectrum shared with the SU2 and SU3. This sharing is done basis on the bidding approach, and the network performance between the SU using the spectrum has been monitored. The communication that exists between various $\mathrm{SU}$ is subject to Miss Detection, and hence the group formation has been done with the Winning SU in a particular PU's. The communication between different clusters SU may subject to a member of losing, where the miss detection probability of communication is higher.

The miss detection and false alarm probability is mitigated with the best selection of SU, and the coalition game theory approach works on with the CSS of SU to allocate the spectrum, this mechanism proposes an energy efficient in CR networks and the spectrum access by SU is done with the idle spectrum in PU in a secured manner without causing interference between them [1]. The Spectrum sensing among the cluster approach between SU is done with a Greedy

\section{Figure 1}

Proposed Model for Winning SU group formation

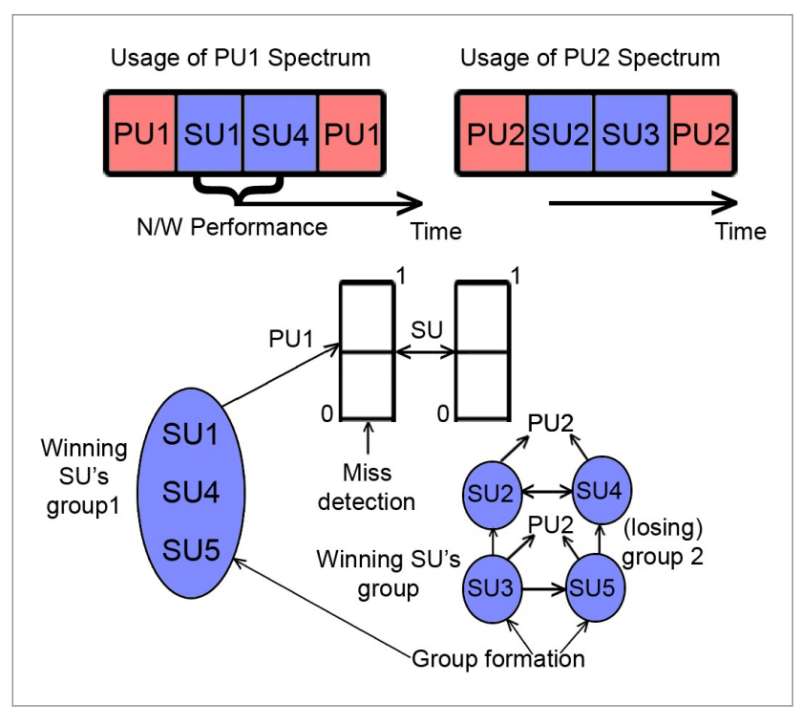

based bidding where the SU compete with their bidding to access the PU idle spectrum. Hence the bidding process is applied with the CSS and the winner $\mathrm{SU}$ is determined based on the authenticated agents in the PU [17]. The spectrum access also follows a rule of incentive based approaches from the bidding, where the non-winner SU to access the spectrum is provided with their rewards back to them for their bidding cost in the CSS paradigm [22]. The CSS paradigm uses an Effective mechanism to apply the Q-learning and Greedy bidding mechanism to enhance energy optimization and the security parameters [7]. The Physical communication between SU and PU often susceptible to various attacks, the main attack which affects the secure communication at the cluster level is eavesdropping attack; it creates interference in PU's [13]. To mitigate this attack a Q-learning approach has been proposed that works on the $\mathrm{SU}$ cluster and the Q-learning algorithm is an action selection policy and agent based algorithm, that handles the rewards for the SU in the current state of being either the spectrum is allocated or not allocated. The state with the corresponding reward for the SU in the cluster is formed with the Q-learning approach [36].

Eavesdropping is one of the most dangerous attacks that happen in the physical communication paradigm in the physical communications segment and it maximizes the interference within the system [37]. The coalition game and Q-learning ensure a higher level 
of security and cooperative communication is performed in the Physical layer level communication [11]. The channel and increased noise rate is also a parameter for the implementation strategy with increased energy conservation [34]. Figure 2 showcase the eavesdropping scenario with the transmission matrix for data transmission in a different scenario with the Eavesdropping scenario, where the source and destination communication happens with PU and SUs. The transmission parameter has been formulated with the matrix, during the transmission phase, the eavesdropping may occur to acquire the transformation parameters information. The direct and indirect transmission may acquire within the $\mathrm{S} 1$ to $\mathrm{Sn}$. The Eavesdropping is more likely to occur within the indirect transmission.

\section{Figure 2}

Eavesdropping Scenario

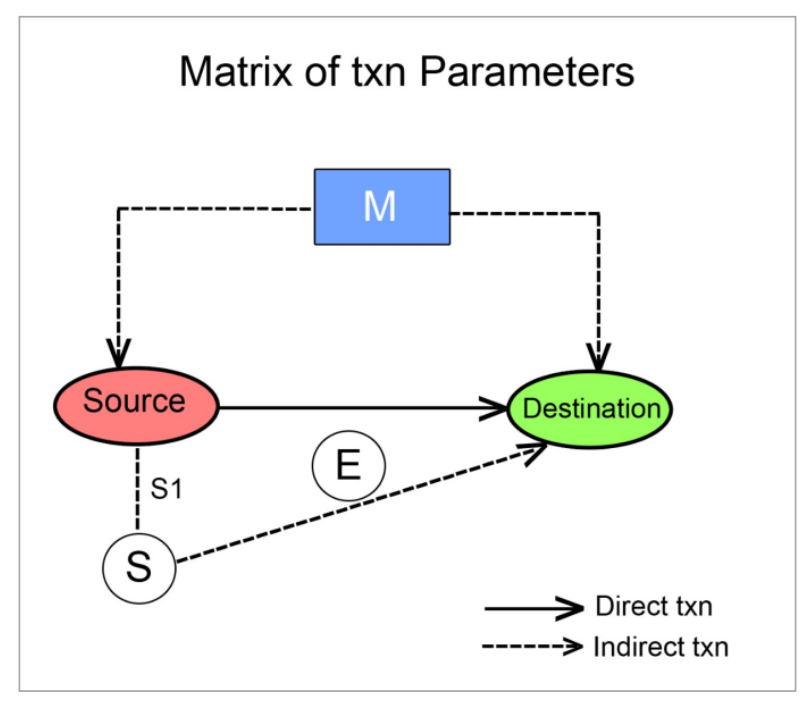

The contribution of the work includes

The CR network performs a SU group formation that has to win SU in a cluster to enhance the spectrum utilization with the usage of Greedy Bidding

The group reformation within the SU cluster to identify the miss detection and false alarm probability has been done after the winner determination from Greedy Bidding. The reformation supports a more enhanced way of spectrum assignment, and it may improve spectrum management and secured communication.
The spectrum access from SU is enhanced with Coalition game formulation and Q-learning approach that performs the energy enhancement and mitigates the Eavesdropping attack along with the identification of the miss detection and false alarm during the communication.

The proposed method is focused with single PU and multiple SU to monitor the network efficiency and SU selfishness to acquire the spectrum in the cluster is measured with the energy enhancement, and the tradeoff between various security parameters are shown with the simulation experiment.

Coalition Game formation is used to enhance the energy conservation in $\mathrm{CR}$ networks and also the attack mitigation is done at the extreme level.

The paper is organized as follows in section 2 presents related works, section3 highlights the system model in that proposed model for game theory formulation and Q-learning approach for enhancing the energy and security in eavesdropping is discussed. Section 4 deals with the simulation results and discussion towards the proposed model, and section 5 focuses the conclusion with future work.

\section{Related Works}

Cognitive radio networks are among those wireless networks, it resolves the spectrum scarcity problem with dynamic spectrum access and to avoid the interferences happening between cognitive users. The two main challenges in CR networks are energy efficiency and secure transmission without attacks in the network. The CR networks are different from other intelligent networks and technologies based on their actions and data flow. It is an adaptable software process, providing access to the transmission parameters and the sensors. The CR network devices provide control and feedback mechanism. The CR networks are further classified into spectrum sensing and full radio based on the transmission and reception parameters [20].

The spectrum management process is categorized into four cadres such as spectrum sensing, spectrum decision, spectrum sharing, and spectrum mobility [12]. Spectrum sensing is the major challenging part of the CR network, where the spectrum allocation 
relies on monitoring the unused portion of the spectrum, to detect available spectrum bands and detect the spectrum holes. The spectrum decision is applied to the CR user to allocate the spectrum based on the channel policy. The spectrum sharing avoids the multiple users allocating the spectrum and accessing the same band or the same spectrum [15]. The spectrum mobility, if a PU needs a particular portion of the spectrum that is in use, it may be allotted with a vacant portion [23]. The above functionalities necessitate significant interactions that spectrum management support cross-layered approach in CR networks.

The cooperative spectrum sensing, the SU sense the channel in a collaborative manner which may enhance the network to be aware of multipath fading, shadowing and penetration loss. The cooperative sensing mitigates the interference between Cognitive users. Cooperative sensing enhances energy consumption, reduces throughput, and delay in the vehicular network [8]. The SU signal has been reported to the fusion centre, and each SU reports a different Signal to Noise Ratio (SNR) for the primary signal. The energy efficiency has been improved in sensing using optimization programming and improves the sensing time using optimization algorithms [25].

A hybrid approach has been implemented on cooperative spectrum sensing, and in this, a collaboration of Energy detection and cyclostationary feature detection with low complexity and high-performance detection has been studied. The individual CR node will decide their energy detector due to their SNR performance in it. A linear classifier has been proposed with the fusion centre it collects the information about the energy monitoring and information sharing in the detected CR nodes [9]. A CR network is an efficient method for spectrum management resources. The spectrum management is performed, and spectrum sensing is restricted with multipath effect and shadow fading due to low probability detection [10]. The proposed method has been considered for five CR nodes. The false alarm and detection probability are being considered here with two merging rules $O R$ and AND rule [42]. The detection probability improves the spectrum efficiency in a profound manner.

The spectrum sensing does the interference to be avoided during the spectrum utilization for the primary user. The detection may be identified with multipath fading, uncertainty issues to mitigate the impact detection performance may be done by uncertainty issues with spatial diversity. The cooperative gain with sensing can be devoted to performance degradation $[16,44]$. The cooperation gain can be achieved in the control channel and data fusion with the overhead. Wang Haijun et al. proposed that the Cognitive radio network is an efficient wireless communication to destroy the inefficiency of spectrum usage. The CR networks focus mainly on the ability to detect the spectrum hole. The low SNR ratio with AND-model, OR-model, counting model, double threshold model has been established in analyzing the sensing techniques $[46,41]$.

A cluster-based approach has been implemented at the fusion centre since it handles a large amount of data and fuzzy-based C-means clustering and it has been decided at energy-based cooperative spectrum sensing has been analyzed. The projection of linear based problems at the data set patterns has been proposed. The Fuzzy C means clustering approach has been made by clustering has a multiple Pus [4]. The proposed technique that the tradeoff between utility and energy conservation is discussed. The energy-efficiency problem is very important in the field of CR network, where the utility is maximized, and the energy consumption is minimized in such a CR network [27]. An improved particle swarm optimization algorithm makes the optimization problem PSO employs a co-evolutionary methodology, and then divide-and-conquer strategy provides an energy efficient feasible solution.

The wireless network is a trend of green communication for next-generation wireless networks. The optimum in this approach is the selection of energy-efficient throughput as a metric for optimizing sensing time and sensors in a deployment [18]. An iterative algorithm has been proposed to obtain the optimality with these two parameters. The low complexity algorithm has been an exhaustive search method when compared to the easy way of complexion. Modelled a Distributed Dynamic Load Balanced Clustering (DDLBC) algorithm. Using this algorithm, a cluster has been formed, and each member in the cluster calculates the cooperative gain, residual energy, distance and sensing cost from the neighbour cluster and performs an optimal cluster. The cluster head has been elected by the cluster member, and through cooperative gain and residual energy the network energy 
consumption enhances channel sensing [19]. In this algorithm, a Markov process model has been formed to reduce the energy consumption in a network. Load-balanced clustering technique $[21,33]$ enhances energy-efficiency and security in ad hoc and cognitive networks. The cluster formation makes the energy-efficiency and accuracy of the channel increased using the proposed algorithm [49].

The spectrum sensing and sharing is a primary challenging task in the CR networks. Various cryptographic algorithms are applied in the security aspects of CR networks in the physical layer with private/public key management and key transmission security in the stack [43]. Security threats may be given from passive eavesdropping nodes that interrupt communications with the authenticated nodes. The CR network has secondary networks, which allocate resources with the strategy proposed in the sensing game to estimate the optimal solution [38]. The secrecy enhancement might develop the optimal solution with the resources, and their maximizing has the CR networks in cooperative jamming to power control and analyze the Dynamic Spectrum Access (DSA). The game players with the uplink of cellular CR networks. The existence of NASH equilibrium in the power control game proposes a strategy with the power control, leads to significantly lower power consumption and a convergence secrecy rate by cooperative gaming [6]. The Chaotic shift keying scheme is proposed to attend the performance of the Rayleigh fading channel. The game theory has a cooperation scheme that has physical layer security that has the primary and secondary transmission of a CR network. The PU leases its own spectrum for the presence of the EavesDropper (ED). The secondary transmitter is a trusted delay with the primary transmitter with a decode and forward fashion to predict the jamming attack. The maximization of the primary secrecy rate and the secondary secrecy rate has been analyzed with the Stackelberg game modelling [3].

The cooperative spectrum sensing in a CR network in which the SU cooperate themselves to detect the Primary user and the possible multiple bands that have been analyzed. The deep cooperative sensing has been proposed with the Convolution neural network to analyze the individual sensing in the training samples [24]. The spectral and spatial correlation of individual sensing with the quantization to propose a
DCS approach within the network. The game-based analysis has been done here with sensing analysis.

The Spectrum sensing is offered a Initial set up of sensing, where the malicious secondary users may enter the network as advertising themselves as the authenticated users. This intrusion may intrude the final outcome also the author proposes a reinforcement learning model to substantiate the working principles of CR networks to analyse the false sensing data. This method proposes a detailed analysis on the adjacent nodes estimation for the agent to merge the high reputation nodes [28]. The former models proposes various game applications but the Proposed solution takes the Q Learning mechanism and Coalition game Modelling to support the security at a greater level with the coalition game modelling

The proposed model forms a cluster with the group formation in the winning SU to frame a reduction mechanism for miss detection and false alarm probability to improvise the communication opportunities [30]. The group spectrum access mechanism is supported by the coalition game formulation and Q-learning-based approach to enhancing the energy with eavesdropping error reduction during the communication paradigm. The proposed model is compared with the Stackelberg and Bayesian game approach[39].

\section{System Model}

Consider a $\mathrm{CR}$ network consists of $\mathrm{M}$ number of PUs that has been labelled from 1 to $\mathrm{M}$ and SU are labelled within 1 to N SU's. Let $\mathrm{R}=\{1, \ldots ., M\}$ and $\mathrm{S}=\{1, \ldots ., N\}$ specifies the associated set of primary users and secondary users. Each PU is considered to be of the licensed channel, and more such licensed channel PU's are available for the shared SU to utilize the idle spectrum from PU. In such cases, the SU is assumed to use the idle channel in the PU. The SU has the transmission parameters in terms of underlay, overlay and interweave. Depending on the spectrum assignment, the SU fixes the PU channel usage, in this paper, the sufficient drive for SU has been considered with the interweave condition when the PU is idle. The miss detection probability hit ratio is measured well using the upper bound limit in the CR network for cluster formation, where the limited number of SU may occupy in a particular cluster is exceeding the limit for 
doing the sensing. The upper bound is a threshold limit assigned for each cluster formation with various secondary users.

Assume the miss detection probability is assumed of in condition limit of $(0 \leq \rho \leq 1)$, where $\rho$ assumes to be miss detection probability parameter estimation. The SU can obtain the transmission parameters. A Rayleigh fading environment is considered for the assumption of the SU parameters, where it subject to identify the signal by SU in the distance node with the transmission parameters. The Rayleigh fading environment is assumed to be of the miss detection and false alarm probability with $p^{\text {missi }}$ having miss detection as ' $\mathrm{i}$ ', and $p^{\text {false }}$ towards the PU as shown in Eq. (1). The assumption is noted as the primary model, where Probability estimation is done with the 1-ex-y(ex-ex!)

$$
\begin{aligned}
& p^{m i s s_{i}}=1-e^{-\frac{\omega}{2}} \sum_{x=0}^{y-2} \frac{1}{x !}\left(\frac{\omega}{2}\right)^{x}-\left(\frac{1+\gamma_{i}}{\gamma_{i}}\right)^{y-1}\left[e^{\frac{-\omega}{2\left(1+\gamma_{i}\right)}-e^{-\omega / 2}}-\right. \\
& \left.e^{-\frac{\omega}{2}} \sum_{x=0}^{y-2} \frac{1}{x !}\left(\frac{\omega \gamma_{i}}{2\left(1+\gamma_{i}\right)}\right)^{x}\right]
\end{aligned}
$$

$$
p^{\text {false }_{i}}=\int_{x=0}^{\omega+2} \frac{\left(x,\left(\frac{\omega}{2}\right)\right)}{x} .
$$

In the above notation, the miss detection is obtained with the Rayleigh fading environment, where $x$ deals with the bandwidth in terms of time and $\omega$ deals with the energy threshold. $\gamma_{i}$ represents the average SNR with respect to the received signal in terms of the PU and SU. The received signal strength with a threshold is measured with $\gamma_{i}$ is measured with the $\gamma_{i}=\frac{T_{i} L_{i}}{\sigma}$, where $T_{i} L_{i}$ is the Gaussian product of the transmission power and the path loss between the PU and SU and the $\sigma$ is the Gaussian noise variance. The $\mathrm{y}-2$ depicts the range of threshold applied with SU in terms of $\mathrm{y}$, and the SNR is assumed to be within this threshold. The $L_{i}=m / d^{\frac{e}{i}}$ path loss probability measured with the distance between PU and SU and the path exponent is monitored with the 'e' and ' $m$ ' is the path loss component. The $\int_{x=0}^{\omega+2} \frac{\left(x,\left(\frac{\omega}{2}\right)\right)}{x}$ is measured with the probable gamma function in terms of measuring the distance with PU and SU, and the false detection is measured with the PU and SU distance as shown in Eq. (2).

\subsection{CSS to Enhance Communication with Winner SU}

The SU has a miss detection probability to PU, and the upper bound limit in the CR network for cluster formation, where the limited number of SU may occupy in a particular cluster is exceeding the limit for doing the sensing. The upper bound is a threshold limit assigned for each cluster formation with various secondary users. This cluster group forms a CSS and the upper bound is specified as ' $U$ ' in SU. This group formation includes a candidate discovery with a similar transmission parameter with the miss detection is set in the upper bound limit. The Set of SU having ' $n$ ' candidates in the group is assumed to be off in the transmission range ' $\mathrm{r}$ '. $r=\sqrt[N]{m} \mathrm{P} / \gamma_{i} \sigma$, where $\mathrm{P}$ is measured as the transmission power for the $\mathrm{SU}$, and the sensing result is achieved to be of the SNR with ' 0 ' decibel. The sensing result and the control channel is assumed to be of the consideration with the SU group formation.

The miss detection probability is analyzed with the SU cluster head formation as the winning SU that metrics the ' $S$ ' as the cluster head and it follows $S \in 2^{\text {n }}$. The AND based fusion rule has been applied to the cluster head, and the application is performed with the winner SU. The miss detection probability for PU and the group 'S' formation with the false alarm to PU. The false alarm and miss detection is predicted with the below notations as shown in Eqs. (3)-(4)

$$
\begin{aligned}
& K_{S}^{\text {miss }}=\prod_{i \in S}\left[P_{i}^{\text {miss }}\left(1-e_{p H}\right)+\left(1-P_{i}^{\text {miss }}\right) e_{p H}\right] \text { (3) } \\
& K_{S}^{\text {false }}=1-\prod_{i \in S}\left[\left(1-P^{\text {false }}\right)\left(1-e_{p H}\right)+P^{\text {false }} e_{p} H,\right. \text { (4) }
\end{aligned}
$$

where the miss detection with the PU is predicted with $K_{S}^{\text {miss }} . e_{p} H$ is the probability for detecting the error in the channel allocation in the cluster with head ' $\mathrm{H}$ ' and the Rayleigh fading is detected with the binary phase-shift keying. The false alarm is predicted with the $K_{S}^{\text {false }}$, where the $e_{p} H$ is the probability for detecting the error in the channel allocation in a cluster with head 'H' as shown in Eq. (5).

$$
e_{p} H=\frac{1}{2\left(1-\sqrt{\frac{\gamma_{i} H}{1+\gamma_{i} H}}\right)},
$$

where $\gamma_{i} H$ shows, the average SNR is allotted with the head and the member of SU. The cluster grouping 
assumes the decrease in $K_{S}^{\text {miss }}$ and $K_{S}^{\text {false }}$. The group formation among SU is 1 and 4 decreases the miss detection probability. The CSS responses that the PU is assumed to be off in the remaining SU is losing. The SUs 1,2,3 and 4 are winning, and the SU 5 is assumed to be in the losing state of the CSS. The sensing report is performed in the fusion centre and stored for the future sensing mechanism.

The maximization of winning SUs and the server establishes the group formation in the SUs in an optimized control with

$$
\max \sum_{i \in N}\left(P_{i}^{\text {miss }} \leq x\right)\left(1-P^{\text {false }}\right)
$$

also the

$$
\begin{aligned}
& \sum_{i \in N}\left(S e_{p i} H=1\right), \quad \forall, i \in \mathrm{N} \\
& \sum_{j \in N} S e_{p i j H} \leq a_{j, k} \forall j, k \in N .
\end{aligned}
$$

The objective function that maximizes the number of winning SU and the communication is established with the indicator in the idle spectrum state. The Eq. (6) and Eq. (7) depicts the model of maximizing the winning SU using the winner Group formation 'S'. The indicator is measured with the idle state using the indicator function with the $P_{i}^{\text {miss }} \leq x$. The condition if winning is obtained in the SU is denoted as $\left(P_{i}^{\text {miss }} \leq x\right)=0$, and the winning $\mathrm{SU}$ is obtained with the $\left(P_{i}^{\text {miss }} \leq x\right)=1$. The head is assumed to be ' 1 ' and ' 0 ' and the group level detection is done with the head value in the probability of obtaining the miss detection. The Eq. (8) shows the false probability condition that prevails in the CR winner SU group 'S', and the Eq. (9) shows the miss detection probability that prevails in the CR winner SU group ' $\mathrm{S}$ '. The below estimation identifies the conditional formulation.

$$
\begin{gathered}
P^{\text {false }}=\prod_{i \in N}\left(1-\left(1-P^{\text {false }}\right) \sum_{j \in N} S e_{p i j H}\right) \\
P_{i}^{\text {miss }}=1-\prod_{i \in N}\left(\left(1-P^{\text {false }}\right) \sum_{j \in N} S e_{p i j H}\right) .
\end{gathered}
$$

The group formation possibility between the SU is assumed to be of the same group $\sum_{i \in N}\left(S e_{p i} H=1\right)$ and the non-linear features is directed with the optimization having the indicator function and the binary variables is followed in the relative case.

\subsubsection{Algorithm 1: Group Formation and Selection of Winning SU}

1. initialize $\forall i \in N, S_{\mathrm{i}}=\{i\}$

2. $\forall i \in$ NidentifiestheneighboursSU with $S U 1,2,3,4$ \&5

3. for all $i \in N d o$

4. if $\mathrm{M} S_{i} \neq \varnothing$ then

5. Optimization $\mathrm{O}_{\mathrm{i}}(\{\mathrm{Si}\}, M)$

6. select $\mathrm{PU}$ with optimized condition

7. else

8. PU is selected according to the $p^{\text {miss }_{i}} \in M$

9. do the process repeatedly

10. Winning $\mathrm{SU}=\left(\{\mathrm{Si}\}, i \in N, \mathrm{M} S_{\mathrm{i}} \neq \varnothing\right)$

11. for all $i \in N d o$

12. if $\mathrm{M} S_{i} \neq \varnothing$ then // Cooperative Spectrum Sensing 13. i collects the $\mathrm{SU}$ from cluster $\mathrm{S}_{\mathrm{j}}$ from $\forall j \in N$

14. $\mathrm{E}_{\mathrm{i}}=\varnothing$

15.

16.

$$
\text { for all }\left[\mathrm{j} \in[m \in N] \mathrm{M} S_{i} \neq \varnothing\right] \text { do }
$$

17. if $\mathrm{MS}_{i} \mathrm{~S}_{\mathrm{j}} \neq \varnothing$ then add i with $S i \cup \mathrm{S}_{\mathrm{j}}$ to $\mathrm{E}_{\mathrm{i}}$

18. if $\mathrm{E}_{\mathrm{i}}=\varnothing$ then //Winner determination 19. i selects the $S i$ and PU by providing optimization 20. $\mathrm{OP}_{\mathrm{i}}\left(\mathrm{E}_{\mathrm{i}}, \mathrm{M}\right) / /$ optimization

21. i performs the group member to collaborate

22. SU forms the selfish group formation with min Error prob

23. else // Losing case

24. i replaces the $S_{\mathrm{i}}$ with $S i \cup \mathrm{S}_{\mathrm{j}}$ to $\mathrm{E}_{\mathrm{i}}$, where $\mathrm{j} \in \mathrm{N}$, $\mathrm{M} S j \neq \varnothing$ and minimizes

25. $\forall j \in N, S_{i}=\{i\}$

26. else

27. Until $S i \cup \mathrm{S}_{\mathrm{j}}$ to $\mathrm{E}_{\mathrm{i}}$ where $\mathrm{j} \in \mathrm{N}, \mathrm{M} S j \neq \varnothing==\mathrm{SU}$ //winning is predicted

The spectrum allocation among different Pus in a CSS identifies a simple idle spectrum based on the situation where the interference is not disturbing the PU communication. The CSS form a cluster group within the process of identifying the complexity and utility within the group schemes. The design of individual SU comprises of the SU group formation and the CSS mechanism, and the selfish group has been fixed with 
the relationship between the PU and SU to converse the optimization.

Each SU identifies the transmission parameters, and hence the communication is established with the parameters in an effective way that the miss detection probability is assumed to follow an optimization in the with PU in $\mathrm{OP}\left(\mathrm{SiS}_{\mathrm{i}}\right)$. The set of SUs formed is grouped together to associate in a manner of forming the optimal association in the groups with SU, and each PU is attained thereof with the return optimal policy prediction as shown in Eq. (10).

\section{$\max _{S i \in S i i \in S M} \operatorname{Tr} S i$,}

(10)

where the transmission parameters are assumed of with the $\mathrm{Tr}$ and the secondary users are measured with the $\max _{S i \in S i} \in S M T r S i$. The set is marked with the $\mathrm{Si}$, and the groups are collaborated to form the ratio of the selection with the SU forming a $j \in \mathrm{N}$. The Objective function is attained with the maximization of the group winning SU as shown in Eq. (11)

$$
\operatorname{TrSi}=\left(1-P U_{\text {Unuse }}\right)\left(1-P_{\text {det }}^{\text {false }}\right) \frac{1}{W_{G}^{\text {group }}} \frac{P U}{\sum_{i, j \in S i} P U^{\text {det }}},
$$

where the unused primary user probability is measured with the spectrum whichever idle in PU with $P U_{\text {unuse }}$ and the PU is assumed to be used with BUSY/IDLE state control. The $P^{\text {false }}$ is the second property where the idle spectrum is assumed to be of the progressive state in the formulation for the PU and the spectrum state is assumed to be of the state for noticing the unused idle spectrum. $\frac{1}{W^{\text {group }}}$ determines the winning secondary group in the CR network with CSS. $\frac{P U^{d e t}}{\sum_{i, j \in S i} P U^{d e t}}$ measures the secondary user identification in the detection capability of the secondary user in the predetermined SU to achieve group reformation with only a secondary group winning strategy. The objective function indicates that the winning SU has the constraint nomination in the maximization of the communication establishment and energy preservation in the CR network.

\subsubsection{Algorithm 2: Group Reformation with Selfishness and Selection of Winning SU for Energy Enhancement}

1. Assume selection of winning group $\mathrm{S}_{\mathrm{i}}$

2. for all $i \in \mathrm{S}_{\mathrm{i}} \mathrm{do}$

3. i performs the group analysis with the members $S_{m}^{i}$ using $\mathrm{OP}\left(\mathrm{SiS}_{\mathrm{i}}\right)$.

4. i communicate to all members for all $j \in \mathrm{S}_{\mathrm{i}}$ of $S_{m}^{i}$

5. $\mathrm{T}=\mathrm{S}$

6. repeat

7. $\quad \mathrm{i} \in \mathrm{T}$

8. if $\forall j \in S_{m}^{i}, S_{m}^{i}==S_{m}^{j}$ then

9. $\quad \forall k \in S_{m}^{i}$ replace $S_{m}^{i}$ withk

10. $\quad \forall m \in \frac{S_{m}^{i}}{S} \operatorname{with}\{m\}$

11. $\quad \mathrm{T}=\mathrm{T} / S_{m}^{i}$

12. else

13. $\mathrm{T}=\mathrm{T} /\{\mathrm{i}\} / /$ Time and energy conservation during CSS

14. Until $\mathrm{T}==\varnothing$

The above group formation concentrates the time and energy conservation during sensing with the SU. The winning SU is assumed to be of the opportunity in the energy conservation for the account of forming the relational SU with PU. The algorithm 1 shows the SU control access with the PU identification and group formation with complexity and overhead prediction. Each SU forms a group with the neighbouring SU and transmits the data in the near group $\mathrm{N}$, and hence the data is assumed to be of in the miss detection probability assumption for the optimization analysis. The sensing lines are formed thereof with the cooperation assumed in the SU. The group updating happens till the winning $\mathrm{SU}$ is judged with the transmission parameters, and the remaining SU are assumed to be losing one in the selfish formation in the SU.

\subsection{Coalition Game Model to Enhance Security and Energy in CSS}

The game model proposed here is the coalition game model to prevent the eavesdropping attack, and the noise is a parameter considered for enhancing the secrecy since the wireless transmission occupies a more discharge of energy in node during the communication. The noise in the interference has an orthog- 
onal assumption that takes place in the winning SU and the CSS formation. The report can be masqueraded in the channel and hence the noise is observed with the attack behaviour. The decision making is considered as artificial noise interference and energy consumption to follow a game order. The game-based assumption is considered with resource modelling with the players having their own actions and strategies to infer a payoff and cost formation. The proposed application is modelled as a game formation with the security as the network resources, players formulate each node, and the noise is accumulated with the strategy. The decision to follow up a game depends on the game movement with the request and the formulation in another strategy as depicted in Table 1 with the assumption parameters.

Table 1

Coalition game for the proposed model

\begin{tabular}{c|l}
\hline Parameters & \multicolumn{1}{c}{ Description } \\
\hline $\mathrm{AC}$ & Players action with cost associated \\
\hline $\mathrm{E}_{\mathrm{AC}}$ & $\begin{array}{l}\text { Energy consumption for Players action } \\
\text { with cost associated }\end{array}$ \\
\hline $\mathrm{E}_{\mathrm{W}}$ & $\begin{array}{l}\text { Energy consumed in SU nodes using } \\
\text { coalition game in weights }\end{array}$ \\
\hline $\mathrm{S}_{\mathrm{W}}$ & $\begin{array}{l}\text { Security enhanced with weight using } \\
\text { coalition game }\end{array}$ \\
\hline $\mathrm{N}$ & Nodes depicted as players \\
\hline $\mathrm{S}$ & Strategy of players with benefit associated \\
\hline
\end{tabular}

The CR networks work on with the awareness of the jamming nodes per channel and the overall performance is assumed to be of in the increasing paradigm with a centralized way. The centralized approach has an impact on the payoffs, and network security gradually decreases the energy conservation parameters. The payoff estimation increases the network with the weighted average of the actions and the strategical game plan. The game has no co-association with the players' payoff and other players' strategy. The calculation of the payoff matrix enables the final assumption in the energy increasing and decreasing with the noise parameters in the channel as depicted in Table 2.

The increase in the security level is assumed to be of in relation to the energy in each SU nodes, noise parameter influences the channel in the prescribed model. The noise causes an interference in the channel that may disturb the node selection and the feasible actions are assumed to be of in the number of available channels depends on the payoff as shown in Eq. (12)

Game Payoff $\mathrm{E}_{\mathrm{i}=} \mathrm{E}_{\mathrm{AC}+} \mathrm{S}_{\mathrm{Wx}} \mathrm{S}$,

where Energy consumption $\left(\mathrm{E}_{\mathrm{W}}\right)$ for Players action with cost associated $\left(\mathrm{E}_{\mathrm{AC}}\right)$ and Energy consumed in SU nodes with 'S' groups and ' $\mathrm{S}_{\mathrm{W}}$ ' winning secondary users using coalition game in weights is equal when both summated to One as shown in Eq. (13)

$$
\mathrm{E}_{\mathrm{W}+} \mathrm{S}_{\mathrm{W}}=1
$$

The game identifies a payoff with the weight distributed in the security and the energy is proportional to

\section{Table 2}

Energy and security matrix representation using payoff in coalition game

\begin{tabular}{l|l|l|l}
\hline \multicolumn{1}{c|}{ Payoff matrix } & \multicolumn{1}{c|}{ No interference } & \multicolumn{1}{c}{$\begin{array}{c}\text { Interference with noise } \\
\text { in allotted channel }\end{array}$} & $\begin{array}{c}\text { Interference with noise } \\
\text { in idle channel }\end{array}$ \\
\hline No interference & Energy and security is same & $\begin{array}{l}\text { Security Increases with constant } \\
\text { energy and decreasing energy }\end{array}$ & $\begin{array}{l}\text { Security Increases with } \\
\text { constant energy }\end{array}$ \\
\hline $\begin{array}{l}\text { Interference with noise in } \\
\text { allotted channel }\end{array}$ & $\begin{array}{l}\text { Security Increases with } \\
\text { constant energy and } \\
\text { decreasing energy }\end{array}$ & $\begin{array}{l}\text { Security Increases with } \\
\text { decreasing energy }\end{array}$ & $\begin{array}{l}\text { Security Increases with } \\
\text { decreasing energy }\end{array}$ \\
\hline $\begin{array}{l}\text { Interference with noise in } \\
\text { idle channel }\end{array}$ & $\begin{array}{l}\text { Security Increases with } \\
\text { constant energy and } \\
\text { decreasing energy }\end{array}$ & $\begin{array}{l}\text { Security Increases with } \\
\text { decreasing energy }\end{array}$ & $\begin{array}{l}\text { Security Increases with } \\
\text { decreasing energy }\end{array}$ \\
\hline
\end{tabular}


the noise parameters. The artificial noise interference issued in accordance with the transmission time is illustrated with the cost of the beneficiary in terms of the cost as shown in Eq. (14)

$$
\mathrm{AC}_{\mathrm{SU} 1}=\mathrm{E}_{\mathrm{W}+} \mathrm{S}_{\mathrm{Wx}} \mathrm{t}_{\mathrm{o}} / \mathrm{T} \text {. }
$$

The cost associated creates a beneficiary that makes sense for the security in the associated model with the number of nodes transmitting in the below noise with the available free channel. This estimation is done for the secondary user 1 in the cluster formation. It has been observed that the maximal node transmission parameters rely on the successful accumulation. The node selected in the channel assumes a cost association in order to generate a higher amount of noise prediction with the decrease in noise. The occupancy of the channel is not increased with the interference increasing. The time $t_{0}$ makes the attacker misinterpreting form data transmission without causing a delay as shown in Eq. (15). The cost is delayed with the several benefits on the transmission delays for the second node that can be simplified as

$$
\mathrm{AC}_{\mathrm{SU} 2}=\left(\mathrm{E}_{\mathrm{W}+} \mathrm{S}_{\mathrm{Wx}} \mathrm{t}_{\mathrm{o}} / \mathrm{T}\right) \times \mathrm{P}_{\mathrm{SU}} \text {. }
$$

$\mathrm{P}_{\mathrm{SU}}$ determines the noise probability within the packets transmitted with these two nodes that have not been overlapped. The Eq. (16) shows the noise probability estimation using the associated cost of SU

$$
\mathrm{P}_{\mathrm{SU}}=1-\mathrm{AC}_{\mathrm{SU} 1}
$$

The above condition is applied for the $\mathrm{N}$ number of nodes to determine the probable network where associated cost of $\mathrm{SU}$ is done with the initial time $t_{0}$ as shown in Eq. (17).

$$
\mathrm{AC}_{\mathrm{SUn}}=\left(\mathrm{E}_{\mathrm{W}+} \mathrm{S}_{\mathrm{WX}} \mathrm{t}_{\mathrm{o}} / \mathrm{T}\right) \times \mathrm{P}_{\text {Sun. }}
$$

Benefits gathered with energy in each node $\mathrm{B}=\mathrm{AC}_{\mathrm{SUn}}$ $/ \mathrm{AC}_{\mathrm{SU} 1}$, where $\mathrm{P}_{\mathrm{Sun}}$ is assumed to be the probability of the non-correlating nodes and the cost associated with each node is predicted based on the benefits of the secondary node usage in the parametric representation that fulfils the security and energy assumption in game modeling technique as shown in Eq. (18).

$$
\mathrm{PS}_{\mathrm{un}=1-} \sum_{i=1}^{N-1} \mathrm{AC}_{\text {Sun. }}
$$

The strategy is associated with the noise interference where the network information is shared between the SU for collaborative sensing each node in the network.

- Each node in CR network uses an application time of weightage in the energy of nodes minus the transmission time is taken. The network lifetime varies according to each node with frequency hopping of the channel.

- Using the game theory model, each SU node transfers the data with selfish opinion irrespective of the neighbours

- The optimization in payoff makes energy conservation with the changing needs of the network with the applied battery source.

- The time $t_{o i}$ is fixed for the travel in the SU node, and the security parameters with the weight in payoff make the channel isle for spectrum utilization.

- The payoff calculation is performed with energy monitoring with the previously stored information, and the collective information with the noise interference is decided in the noise interference channel. The evaluation results showcase that the results have been enriched with security enhancement.

The eavesdropping has been monitored with the assumptions based on the multiple channels with the spectrum sensing if the channel has not been identified for channel sensing it will start the process from beginning to assume the data within the node parameters. The proposed method uses two monitoring trends with the free channel and random channel for security detection.

\subsection{Reinforcement Learning with Q-learning Model to Enhance Energy Model}

The Q-learning model estimates the approach with the off-policy control algorithm where the policy agent is used to update the following condition for making the cluster head to be applied to the behaviour environment [23]. The time interval for the measured environment is observed by the knowledge of each agent and the Q-learning for the knowledge measured is denoted by the 


$$
\begin{aligned}
& Q_{n+1}^{\mathrm{a}}\left(s_{n}^{\mathrm{a}}, a_{n}^{\mathrm{a}}\right)=(1-€) Q_{n}^{a}\left(s_{n}^{\mathrm{a}}, T_{n}^{\mathrm{a}}\right)+€\left[r_{n+1}^{\mathrm{a}}\left(s_{n+1}^{\mathrm{a}}\right)+\right. \\
& \left.¥ \max _{a \in A} Q_{n}^{\mathrm{a}}\left(s_{n+1}^{\mathrm{a}}, X\right)\right],
\end{aligned}
$$

where $S_{n}^{\mathrm{a}}$ represents the state of agent a at time t, $a_{n}^{\mathrm{a}}$ represents action, $r_{n+1}^{\mathrm{a}}\left(s_{n+1}^{\mathrm{a}}\right)$ represents delayed rewards for the action taken at time $\mathrm{n}$ and receives at time $\mathrm{n}+1$. The learning rate I predicted with the $€(0<€<1)$ is the learning rate and $(0<¥<1)$ represents the cut off factor as shown in Eq. (19).

- Initialize the Primary CR network with $Q_{1}$ and target CR network with $Q_{n}$

- The Buffer in the CR network $\mathrm{i}$ assumed to be of B with the time limit $t<<1$

- For each iteration do

- For each operating environment do

- Examine the state $s_{n}^{\mathrm{a}}$ and select $\mathrm{g}_{\mathrm{n}} \sim \pi\left(\mathrm{g}_{\mathrm{n}}, \mathrm{s}_{\mathrm{n}}\right)$

- Execute $g_{n}$ and predict the next state $s_{n}^{\mathrm{a}}+1$

- Reward $\mathrm{r}_{\mathrm{n}}=\mathrm{R}_{\mathrm{w}}\left(s_{n}^{\mathrm{a}}, \mathrm{g}_{\mathrm{n}}\right)$

- Store $\left(s_{n}^{\mathrm{a}}, \mathrm{g}_{\mathrm{n}}, \mathrm{R}_{\mathrm{w}}, s_{n+1}^{\mathrm{a}}\right)$ in the primary network

- Store in buffer B

- For each update step do

- SampleSP $\mathrm{n}_{\mathrm{n}}=\left(s_{n}^{\mathrm{a}}, \mathrm{g}_{\mathrm{n}}, \mathrm{R}_{\mathrm{W}}, s_{n+1}^{\mathrm{a}}\right) \sim \mathrm{B}$

- Predict the target in CR network with $Q_{n}$ Value

- Perform gradient descent step on $\left(\mathrm{Q}_{1}\left(s_{n}^{\mathrm{a}}, \mathrm{g}_{\mathrm{n}}\right)\right.$ $\left.\mathrm{Q}_{\mathrm{n}}\left(S_{n}^{\mathrm{a}}, \mathrm{g}_{\mathrm{n}}\right)\right)$

- Update target network parameter $Q_{n} \leftarrow t^{*} Q_{1}(1-t)$ ${ }^{*} \mathrm{Q}_{\mathrm{n}}$

- Strategy with payoff $Q_{n} \leftarrow t^{*} Q_{1}(1-t) * Q_{n} /$ SUn // payoff to enhance energy and security.

The strategy supports the modelling phase with the game modelling to enhance each $\mathrm{SU}$ with the $\mathrm{Q}$ based approach that enhances the payoff to increase the energy and eavesdropping in the CR network.

\section{Results and Discussion}

This section presents the results obtained in the experimental study with the proposed strategy. The simulations have been carried in CWSN simulator, and the work focuses mainly on the physical layer. The values of $\mathrm{N}, \mathrm{SU}, \mathrm{PU}, \mathrm{d}$ are all determined with the miss detection, and false alarm probability count and the transmission ranges are assumed to be for a different number of cluster SUs. The simulation parameters are discussed below in Table 3. Consider a simulation area with the 70 nodes been taken in to consideration as secondary users. The frequency band is used with the $700 \mathrm{MHZ}$ and the transmission power is maintained at the 100MW. The probability is analysed with the false alarm and miss detection ratio. The time parameter is assumed to be of $\mathrm{T}$ with the 0.3 to 0.5 seconds. The energy utilised with the respect to the secondary user is assumed to be of the ratio in the winning secondary users and consolidated with the alarm probability with 0.7 respectively as depicted in Table 3 .

Table 3

Simulation parameters

\begin{tabular}{l|l}
\hline \multicolumn{1}{c}{ Parameter } & \multicolumn{1}{c}{ Values } \\
\hline Simulation Area & $250 \mathrm{X} 250 \mathrm{~m}$ \\
\hline Frequency band & $700 \mathrm{MHz}$ \\
\hline N- Number of SU nodes & 70 nodes \\
\hline Transmission power of SU & $15 \mathrm{~mW}$ \\
\hline Transmission power of PU & $100 \mathrm{~mW}$ \\
\hline Gaussian Noise & $-80 \mathrm{dbm}$ \\
\hline Path loss exponent & 3 \\
\hline Threshold energy & $15 \mathrm{~mW}$ \\
\hline False alarm probability & 0.0 .14 \\
\hline Miss detection probability & 0.03 \\
\hline $\mathrm{T}$ & $0.3-0.5 \mathrm{~s}$ \\
\hline Number of channels & 25 \\
\hline $\mathrm{E}_{\mathrm{W}}$ & 0.3 \\
\hline $\mathrm{S}_{\mathrm{W}}$ & 0.7 \\
\hline
\end{tabular}

Figure 3 illustrates the relationship between the number of Secondary users $\mathrm{N}$ and the ratio of winning SU with the proposed mechanism. The proposed model uses the selfish group formation and reformation to support the CSS formation to enhance energy and security. The proposed model 
uses the coalition game modelling. It also has been compared with the non-cooperative model and recall model. The proposed mechanism outlines that the performance enhances when the number of SU becomes large with $\mathrm{N}>30$. The performance ratio is $2 \%$ when it reaches with $\mathrm{N}=50$. This detection is obtained with the miss detection and probability of false alarm in the winning groups in $\mathrm{N}=50$. The miss detection probability in the proposed mechanism is achieved with 0.02and the SU tries to form a reformation group that eradicates the miss detection probability. The proposed mechanism has a $15 \%$ lower than the rest methods of recall model, where the model is reframed with the existing parameters of transmission, but it does not enhance the miss detection probability and the false alarm probability. The model has been compared with the Stackelberg and Bayesian game modelling, whereas the proposed coalition game modelling provides the best detection probability compared with other models.

\section{Figure 3}

Group formation in winning SU and Optimal SU for Miss detection and False alarm prediction

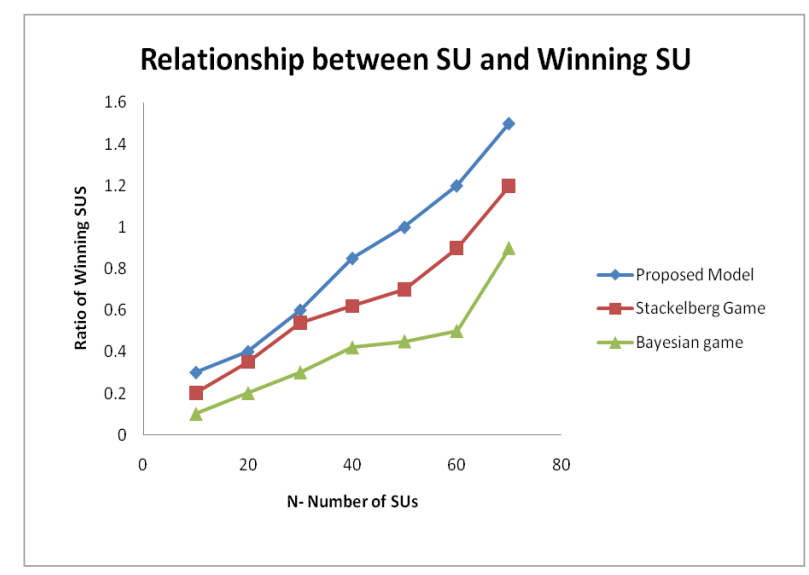

Figure 4 represents the energy spent with the proposed model and Stackelberg game. At time t-0.1 the communication is observed with the secrecy rate energy conservation. Energy consumed in SU nodes using coalition game in weights is assumed as ' 0 ' initially, and then it has been assumed as 0.8 as shown in figure 5 . The results describe that the proposed model eradicates the miss detection in a busy channel with the eavesdropping and legitimate nodes have been identified.
The proposed model enhances the energy, and the security is enhanced since the energy spent is maximum in proposed, but the consumption is maintained with the model that supports the secrecy communication in a predetermined way. The model has been compared with the Stackelberg and Bayesian game modelling, whereas the proposed coalition game modelling provides the best energy enhancement. The energy consumed is more in the proposed model while comparing with the other two game modelling approaches. The table below depicts the purpose of adding a detailed prediction of energy with the Q-learning approach.

\section{Table 4}

Energy Consumed based on the energy spent with the $\mathrm{E}_{\mathrm{W}}=0$ \& 0.7

\begin{tabular}{c|c|c|c}
$\begin{array}{c}\text { Energy } \\
\text { Consumed } \\
(\mathrm{J})\end{array}$ & $\begin{array}{c}\text { Coalition game } \\
\text { with CSS at time } \\
\mathrm{t}=0.1\end{array}$ & $\begin{array}{c}\text { Stackelberg } \\
\text { game at } \\
\text { time } \mathrm{t}=0.1\end{array}$ & $\begin{array}{c}\text { Bayesian } \\
\text { game at time } \\
\mathrm{t}=0.1\end{array}$ \\
\hline 0.1 & 1.1 & 0.9 & 0.7 \\
\hline 0.2 & 1.3 & 1 & 0.9 \\
\hline 0.3 & 1.6 & 1.4 & 1.2 \\
\hline 0.4 & 1.8 & 1.5 & 1.3 \\
\hline 0.5 & 1.9 & 1.6 & 1.5 \\
\hline 0.6 & 2 & 1.9 & 1.7 \\
\hline 0.7 & 2.5 & 2.2 & 2 \\
\hline 0.8 & 2.7 & 2.5 & 2.3 \\
\hline 0.9 & 2.9 & 2.6 & 2.4 \\
\hline
\end{tabular}

\section{Figure 4}

Energy spent with $\mathrm{E}_{\mathrm{W}}=0$

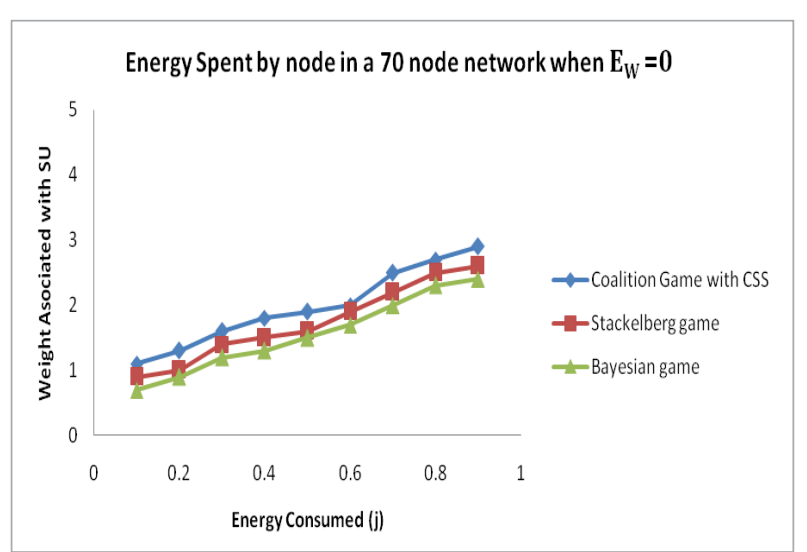


Figure 5

Energy spent with $\mathrm{E}_{\mathrm{W}}=0.7$

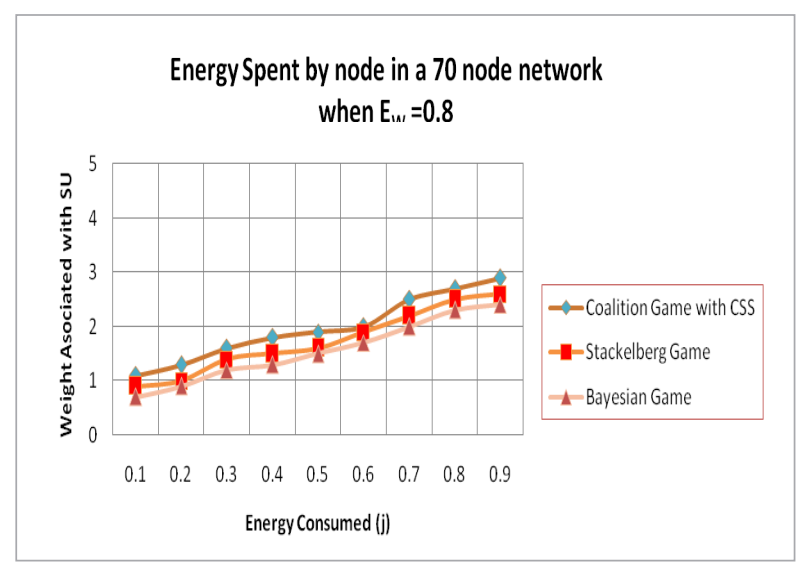

Figure 6 shows that the energy spent using Q-learning, where more energy has been spent but the energy consumed is at the maximum level in the CR network. At time $t=3000$ s the packets sent, and energy usage is moderate but the nodes alive when it reaches the time $\mathrm{t}=-6000 \mathrm{~s}$ depicts that the packet transmission is low but the energy consumed is high. The probability that the energy consumption and the tradeoff between the security and energy is maintained throughout in CR network. The overhead is measured with energy consumption and spectrum utilization. The strategy has been assumed to be of the with the proposed model is higher. The simulations after time $t=6000$ s are shown

\section{Table 5}

Spectrum utilization using Q-learning

\begin{tabular}{l|c|c}
\hline $\begin{array}{c}\text { Energy and spectrum } \\
\text { monitoring }\end{array}$ & $\begin{array}{c}\text { Coalition Strategy } \\
\text { with Q-learning } \\
\text { using battery with } \\
50 \%\end{array}$ & $\begin{array}{c}\text { Non Coalition } \\
\text { strategy using } \\
\text { battery with } \\
50 \%\end{array}$ \\
\hline Energy Spent(j) & 55.2 & 52.1 \\
\hline Energy Consumption & 49.1 & 47.2 \\
\hline $\begin{array}{l}\text { Spectrum utilization } \\
\text { packets sent }\end{array}$ & 55678 & 50567 \\
\hline
\end{tabular}

The above Table 5 depicts that the energy consumption is higher when the node is used with the proposed security assumptions. The proposed model outlines a $26 \%$ increase in the battery level, and then weight / cost associated may not increase the battery nodes to consume less. The spectrum utilization is higher for the proposed model with the non-game strategy. The network lifetime with overhead is achievable in the proposed model.

\section{Figure 6}

Energy spent with different security strategies

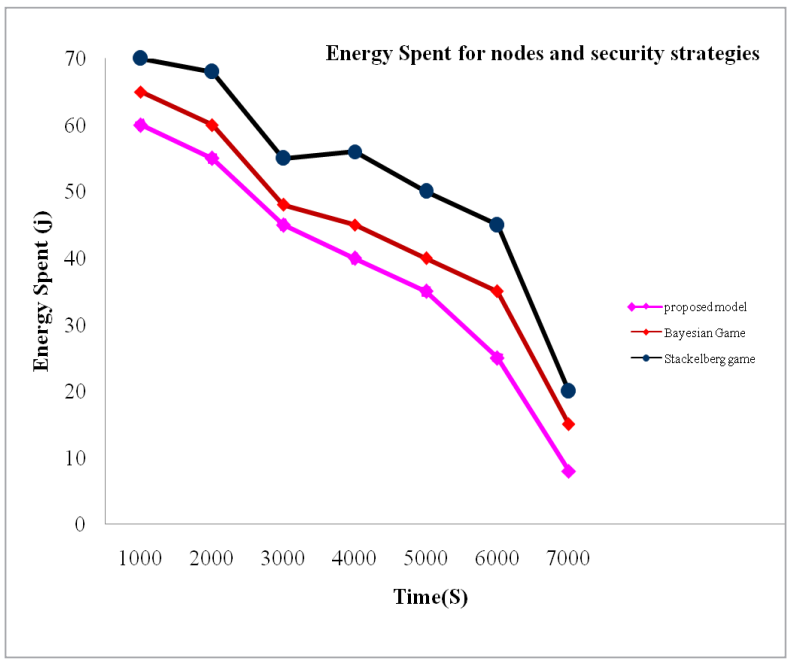

Figure 7 showcase the miss detection probability using the Q-learning approach and the energy consumed is more in Q-learning approach since the probability ratio and the packet delivery ratio gradually increases the proposed approach and the number of

Figure 7

Miss Detection probability in terms of packet delivery ratio

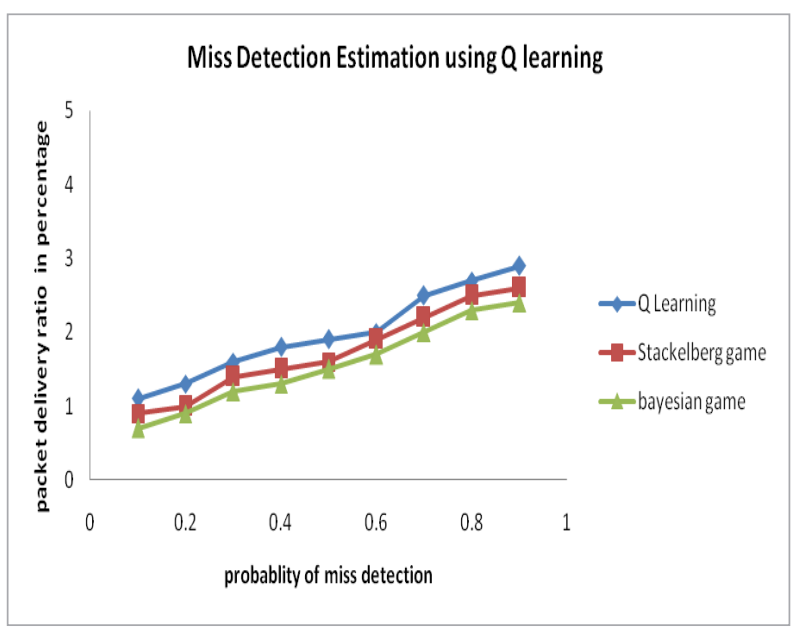


SU nodes is increased with the proposed approach for deliberately improved in the ratio of theenergy with the secured transmission. Figure 8 shows the false alarm probability in the proposed has a closer observation in the proposed approach, and the Q-learning is compared with the Bayesian and Stackelberg game approach .since the proposed model has a probabilistic view with the increase in energy consumption, and it gradually increased.

\section{Figure 8}

False Alarm probability Using Proposed Model

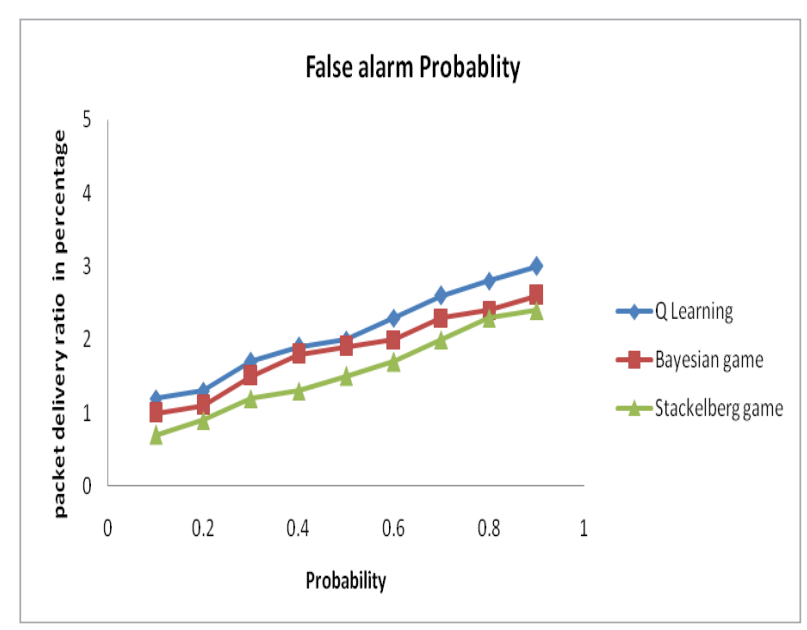

\section{References}

1. Abuzainab, N., Vinnakota, S. R., Touati, C. Coalition Formation Game for Cooperative Cognitive Radio Using Gibbs Sampling, ArXiv, 2014. https://doi.org/10.1109/ WCNC.2015.7127595

2. Afghah, F., Costa, M., Razi, A., Ephremides, A. A Reputation-Based Stackelberg Game Approach for Spectrum Sharing with Cognitive Cooperation. Proceedings of IEEE CDC, 2013, 3287-3292. https://doi.org/10.1109/ CDC.2013.6760385

3. Ali, M. N., Hasan, A.-T. Enhancing Physical Layer Security in Cognitive Radio Networks. Informatica, London, 2016.

4. Anal,P., Santi M.P. Kernel Fuzzy C-Means Clustering on Energy Detection Based Cooperative Spectrum Sensing. Digital Communications and Networks, 2016, 2(4), 196-205. https://doi.org/10.1016/j.dcan.2016.09.002

\section{Conclusion}

In this paper, the spectrum sensing has been investigated with the collaborative manner on supporting the energy enhancement in multiple PUs. The objective function has been obtained with the maximum energy conservation with the winning SU and transmission strategies. The optimization has been performed with the objective function to monitor the network performance and secure transmission with selfish group formation. Through the simulation study, the proposed model increases the winning SU ratio with $2 \%$ and average miss detection probability with $2 \%$ compared to the various models. The throughput is also enhanced with the network performance in the winning SU strategy, the coalition game model enhances energy modelling, and Q-learning supports the game modelling in detecting the eavesdropping to make the channel to effectively allocate the SU spectrum allocation with $7 \%$ of attack modelling. The network performance is modelled with the physical layer contributing to the energy consumption level, and spectrum saturation levels are maintained. In future works, it has been used in multiple channels with the routing protocol to monitor the security and energy conservation with various PU activities also the multiple channel resource allocation can be provided using the proposed model.

5. Beres, E., Adve, R. Optimal Relay-Subset Selection and Time-Allocation in Decode-and-Forward Cooperative Networks. IEEE Trans-actions on Wireless Communications, 2010, 9(7), 2145-2155. https://doi.org/10.1109/ TWC.2010.07.081669

6. Bernardo, F., Agustí, R., Pérez-Romero, J., Sallent, O. Distributed Spectrum Management Based on Reinforcement Learning. In Proceedings of the 4th International Conference on Cognitive Radio Oriented Wireless Networks and Communications, 2009, 1-6. https:// doi.org/10.1109/CROWNCOM.2009.5189161

7. Bremaud, P. Markov Chains Gibbs Fields Monte Carlo Simulation and Queues, Springer, 1999. https://doi. org/10.1007/9r78-1-47577-3124-8

8. Chen, S., Vuyyuru, R., Altintas, O., Wyglinski, A. M. On Optimizing Vehicular Dynamic Spectrum Access Net- 
works: Automation and Learning in Mobile Wireless Environments. In Proceedings of the IEEE Vehicular Networking Conference (VNC '11), 2011, 39-46. https:// doi.org/10.1109/VNC.2011.6117122

9. Chengta, H., Kunqi, G., Sun, L. Efficient Cooperative Spectrum Sensing Methods for Cognitive Radio Networks, Fourth International Conference on Digital Manufacturing and Automation (ICDMA), 2013, 1-9.

10. Chowdhury, K., Doost-Mohammady, R., Meleis, W., Di Felice, M., Bononi, L. Cooperation and Communication in Cognitive Radio Networks Based on TV Spectrum Experiments. In Proceedings of the IEEE International Symposium on a World of Wireless, Mobile and Multimedia Networks (WoWMoM '11), 2011, 1-9. https://doi. org/10.1109/WoWMoM.2011.5986378

11. Coucheney, P., Touati, C., Gaujal, B. Selection of Efficient Pure Strategies in Allocation Games. Proceedings of International Conference on Game Theory for Networks, 2009, 658-666. https://doi.org/10.1109/GAMENETS.2009.5137457

12. Elkashlan, M., Wang, L., Duong, T. Q, Karagiannidis, G. K, Nallanathan, A. On the Security of Cognitive Radio Networks. IEEE Transactions of Vehicular Technology, 2015, 64(8), 3790-3795. https://doi.org/10.1109/ TVT.2014.2358624

13. Geetha, R., Sivasubramanian, S., Kaliappan, M., Vimal, S., Annamalai, S. Cervical Cancer Identification with Synthetic Minority Oversampling Technique and PCA Analysis using Random Forest Classifier. Journal of Medical Systems, 2019, 43, 286. https://doi.org/10.1007/ s10916-019-1402-6

14. Hao, X., Cheung, M. H., Wong, V. W. S., Leung, V. C. M. A Stackelberg Game for Cooperative Transmission and Random Access in Cognitive Radio Networks. Proceedings of IEEE PIMRC, 2011, 411-416.

15. Harold Robinson, Y., Golden Julie, E. MTPKM: Multipart Trust Based Public Key Management Technique to Reduce Security Vulnerability in Mobile Ad-Hoc Networks. Wireless Personal Communication, 2019, 109, 739-760. https://doi.org/10.1007/s112777-019-06588-4

16. Ian, F., Brandon, A., Lo, F., Balakrishnan, R. Cooperative Spectrum Sensing in Cognitive Radio Networks: A Survey. Physical Communication, 2011, 04(01), 40-62. https://doi.org/10.1016/j.phycom.2010.12.003

17. Ilango, S. S., Vimal, S., Kaliappan, M., Subbulakshmi, P. Optimization Using Artificial Bee Colony Based Clustering Approach for Big Data. Cluster Computing, 2018. https://doi.org/10.100r7/s10586-017-1571-3

18. Jiang, T., Grace, D., Liu, Y. Two-Stage Reinforcement-Learning-Based Cognitive Radio with Exploration Control. IET Communications, 2011, 5, 644-651. https://doi.org/10.1049/iet-com.2009.0803
19. Jiang, T., Grace, D., Mitchell, P. D. Efficient Exploration in Reinforcement Learning-Based Cognitive Radio Spectrum Sharing. IET Communications, 2011, 5(10), 1309-1317. https://doi.org/10.1049/iet-com.2010.0258

20. Jouini, W., Ernst, D., Moy, C., Palicot, J. Upper Confidence Bound Based Decision-Making Strategies and Dynamic Spectrum Access. In Proceedings of the IEEE International Conference on Communications (ICC '10), 2009, 1-5. https://doi.org/10.1109/ICC.2010.5502014

21. Kaliappan, M., Mariappan, E., Viju Prakash, M., Paramasivan, B. Load Balanced Clustering Technique in MANET Using Genetic Algorithms. Defence Science Journal, 2016, 66(3), 251-258. https://doi.org/10.14429/dsj.66.9205

22. Kannan, N., Sivasubramanian, S., Kaliappan, M. Vimal.s, A. Suresh. Predictive Big Data Analytic on Demonetization Data Using Support Vector Machine. Cluster Computing, 2018. https://doi.org/10.1007/s10586-018-2384-8

23. Khatta, A., Perkins, D. Magdy, B. Cognitive Radio Networks from Theory to Practice. Springer, 2013.

24. Kok, J. R., Vlassis, N. Collaborative Multiagent Reinforcement Learning by Payoff Propagation. Journal of Machine Learning Research, 7, 2006, 1789-1828.

25. Kun, Z., Pawelcza, P., Cabric, D. Reputation-based Cooperative Spectrum Sensing with Trusted Nodes Assistance. IEEE Communications Letters, 2010, 10897798.

26. Mariappan, E., Kaliappan, M., Vimal. S. Energy Efficient Routing Protocol Using Grover's Searching Algorithm Using MANET. Asian Journal of Information Technology, 2016, 15(24).

27. Meiqin, T., Yalin, X. Energy-Efficient Power Allocation in Cognitive Radio Network Using Coevolution Chaotic Particle Swarm Optimization. Computer Networks, 2016, 100(1), 1-11. https://doi.org/10.1016/j.comnet.2016.02.010

28. Mengbo, Z., Lunwen, W., Yanqing, F. Distributed Cooperative Spectrum Sensing Based on Reinforcement Learning in Cognitive Radio Networks. AEU - International Journal of Electronics and Communications, 2018, 94, 359-366. https://doi.org/10.1016/j. aeue.2018.07.029

29. Monderer, D., Shapley, L. S. Potential Games. Games and Economic Behavior, 1996, 14, 124-143. https://doi. org/10.1006/game.1996.0044

30. Reddy, Y. B. Detecting Primary Signals for Efficient Utilization of Spectrum Using Q-Learning. In Proceedings of the International Conference on Information Technology: New Generations, 2008, 360-365. https://doi. org/10.1109/ITNG.2008.95

31. Saad, W., Han, Z., Debbah, M., Hjorungnes, A. Coalitional Games for Distributed Collaborative Spectrum Sensing in Cognitive Radio Networks. Proceedings of IEEE 
INFOCOM, 2009, 2114-2122. https://doi.org/10.1109/ INFCOM.2009.5062135

32. Saad, W., Han, Z., Zheng, R., Hjrungnes, A., Bassar, T., Poor, H. V. Coalitional Games in Partition Form for Joint Spectrum Sensing and Access in Cognitive Radio Networks. IEEE Journal on Selected Areas of Signal Processing, 2012, 6(2), 195-209. https://doi.org/10.1109/ JSTSP.2011.2175699

33. Sivakumar, G., Kaliappan, M., Jerart Julus, L. Enhancing the Performance of MANET Using EESCP. Proceedings of International Conference on Pattern Recognition, Informatics and Medical Engineering, 2012, 221-230. https://doi.org/10.1109/ICPRIME.2012.6208348

34. Sivaram, M., Kaliappan, M., Shobana, Prakash, M. V., Porkodi, V., Vijayalakshmi, K., Vimal, S., Suresh, A. Secure Storage Allocation Scheme Using the Fuzzy Based Heuristic Algorithm for the Cloud. Journal of Ambient Intelligence and Human Computing, 2020. https://doi. org/10.1007/s12652-020-02082-z

35. Sudha, S., Jerart Julus, L., Vimal, S., Performance of CE-MSK-OFDM for long haul optical transmission, 2014 International Conference on Circuits, Power and Computing Technologies, 2014, 1146-1152. https://doi. org/10.1109/ICCPCT.2014.7054832

36. Suresh, A., Udendhran, R., Vimal, S. An Intelligent Grid Network Based on Cloud Computing Infrastructures. Novel Practices and Trends in Grid and Cloud Computing, 2019, 59-73. https://doi.org/10.4018/978-1-52259023-1.ch005

37. Suresh, A., Udendhran, R., Vimal, S. Cloud-Based Predictive Maintenance and Machine Monitoring for Intelligent Manufacturing for Automobile Industry. Novel Practices and Trends in Grid and Cloud Computing, 2019, 74-81. https://doi.org/10.4018/978-1-5225-9023-1.ch006

38. Thathachar, M. A. L., Sastry, P. S. Varieties of Learning Automata: An Overview. IEEE Transactions on Systems, Man, and Cybernetics B, 32(6), 2002, 711-722. https://doi.org/10.1109/TSMCB.2002.1049606

39. Vimal, S., Suresh, A., Subbulakshmi, P., Pradeepa ,S., Kaliappan, M. Edge Computing-Based Intrusion Detection System for Smart Cities Development Using IoT in Urban Areas. In: Kanagachidambaresan, G., Maheswar, R., Manikandan, V., Ramakrishnan, K. (Eds.) Internet of Things in Smart Technologies for Sustainable Urban Development. EAI/Springer Innovations in Communication and Computing, 2020. https://doi. org/10.1007/978-3-030-34328-6_14
40. Vimal, S., Kalaivani, L., Kaliappan, M. Collaborative Approach on Mitigating Spectrum Sensing Data Hijack Attack and Dynamic Spectrum Allocation Based on CASG Modelling in Wireless Cognitive Radio Networks. Cluster Computing, 2017. https://doi.org/10.1007/s10586-0171092-0

41. Vimal, S., Kalaivani, L., Kaliappan, M., Suresh, A., Xiao-Zhi, G., Varatharajan, R. Development of Secured Data Transmission Using Machine Learning Based Discrete Time Partial Observed Markov Model and Energy Optimization in Cognitive Radio Networks. Neural Computing \& Application, 2018. https://doi.org/10.1007//s00521-018-3788-3

42. Vimal, S., Kaliappan, M., Suresh, A., Subbulakshmi, P., Sanjee, K., Dinesh, K. Development of Cloud Integrated Internet of Things Based Intruder Detection System. Journal of Computational and Theoretical Nanoscience, 2018, 15(11), 3565-35r70. https://doi.org/10.1166/ jctn.2018.7665

43. Vimal, S., Khari, M., Crespo, R. G., Kalaivani, L., Nilanjan, D., Kaliappan, M. Energy enhancement Using Multi Objective Ant Colony Optimization with Double Q Learning Algorithm for IoT Based Cognitive Radio Networks. Computer Communications, 2020, 154, 481490. https://doi.org/10.1016/j.comcom.2020.03.004

44. Vimal, S., Khari, M., Dey, N., Crespo, R. G., Robinson, Y.H. Enhanced Resource Allocation in Mobile Edge Computing Using Reinforcement Learning Based MOACO Algorithm for IIOT. Computer Communications, 2020, 151, 355-364. https://doi.org/10.1016/j.comcom.2020.01.018

45. Vimal, S. et al. Secure Data Packet Transmission in MANET Using Enhanced Identity-Based Cryptography. International Journal of New Technologies in Science and Engineering, 2016, 3(12), 35-42.

46. Wan, H., Su, X., Jing, W. Cooperative Spectrum Sensing Techniques in Cognitive Radio. ZTE Communications, 2009, 2(1), 1-10.

47. Wang, B., Liu, K. J. R., Clancy, T. C. Evolutionary Game Framework for Behavior Dynamics in Cooperative Spectrum Sensing. Proceedings of the IEEE GLOBECOM,2008, 1-5. https://doi.org/10.1109/GLOCOM.2008.ECP.599

48. Wang, X., Ma, K., Han, Q., Liu, Z., Guan, X. Pricing-Based Spectrum Leasing in Cognitive Radio Networks. IET Networks, 2012, 1(3), 116-125. https://doi.org/10.1049/ iet-net.2012.0149

49. Zongsheng, Q., Jinlong, W. Optimal Energy-Efficient Cooperative Spectrum Sensing in Cognitive Radio Networks. Radio Engineering, 2013, 22(4), 1150-1155. 